\title{
Axisymmetric Finite Element Modelling of the Human Lens Complex under Cataract Surgery
}

\author{
M. T. Cardoso ${ }^{1}$, B. Feijóo ${ }^{2}$, A. P. G. Castro ${ }^{1, * \mathbb{D}}$, F. J. Ribeiro ${ }^{2,3}$ and P. R. Fernandes $^{1}$ (D) \\ 1 IDMEC, Instituto Superior Técnico, Universidade de Lisboa, 1049-001 Lisbon, Portugal; \\ teresaaslcardoso@tecnico.ulisboa.pt (M.T.C.); paulo.rui.fernandes@tecnico.ulisboa.pt (P.R.F.) \\ 2 Departamento de Oftalmologia, Hospital da Luz, 1500-650 Lisbon, Portugal; bfeijoo@hospitaldaluz.pt (B.F.); \\ fjribeiro@hospitaldaluz.pt (F.J.R.) \\ 3 Faculdade de Medicina, Universidade de Lisboa, 1649-028 Lisbon, Portugal \\ * Correspondence: andre.castro@tecnico.ulisboa.pt
}

check for updates

Citation: Cardoso, M.T.; Feijóo, B.;

Castro, A.P.G.; Ribeiro, F.J.;

Fernandes, P.R. Axisymmetric Finite Element Modelling of the Human Lens Complex under Cataract Surgery. Symmetry 2021, 13, 696. https://doi.org/10.3390/sym13040696

Academic Editor: Piotr Morasiewicz

Received: 30 March 2021

Accepted: 13 April 2021

Published: 16 April 2021

Publisher's Note: MDPI stays neutral with regard to jurisdictional claims in published maps and institutional affiliations.

Copyright: (c) 2021 by the authors. Licensee MDPI, Basel, Switzerland. This article is an open access article distributed under the terms and conditions of the Creative Commons Attribution (CC BY) license (https:// creativecommons.org/licenses/by/ $4.0 /)$.

\begin{abstract}
Cataract is a disease opacifying the crystalline, leading to a blurred vision and ultimately to blindness. With an aging population, the incidence of cataract is increasing, as well as the number of treatments. The solution available is its complete removal, followed by an implant of an intraocular lens (IOL). Although the post-operative complications on cataract surgeries have been decreasing in general, the bag-IOL complex dislocation is still an issue, probably being the most serious complication under this procedure. In this work, an axisymmetric Finite Element (FE) modelling strategy of the crystalline complex during the process of accommodation under cataract surgery is proposed. The goal was to understand the influence of biomechanical alterations promoted by the IOL on bag-IOL dislocation after surgery. An increase of force and stress in the zonules was verified in the pseudophakic eye compared to the complete eye, which could explain why zonules break years after surgery, leading to the bag-IOL dislocation. The axisymmetric FE model proposed in this work is innovative in this field, which still lacks detailed research, and can be an important complement for the clinical and biomechanical work on the crystalline complex.
\end{abstract}

Keywords: intraocular lens; capsular bag; zonular fibers; pseudophakic eye; axisymmetric finite element modelling

\section{Introduction}

Cataract is a disease that leads to visual impairment, and eventually to blindness, worldwide. With an aging population, the incidence and prevalence of cataract is increasing, as well as the number of treatments. Prokofyeva and co-workers reported that the prevalence of this disease in Europe increased from 5\% of the population between 52 and 62 years-old and from 30\% for 60-69 years old to $64 \%$ in population above 70 years-old (between 2000 and 2009) [1]. More recent studies by Flaxman and co-workers and Bikbov and co-workers have not only confirmed this tendency, but forecasted further growth of cases [2,3]. Cataract surgery with intraocular lens (IOL) implantation has been quickly evolving over the years, with the development of new technology and techniques, such as phacoemulsification and new materials for IOL, making it a safer and more reliable procedure [4-6]. Following cataract surgery, few complications can appear, one of the most serious being in-the-bag-IOL dislocation [7]. This complication arises from a progressive zonular dehiscence associated with contraction of the capsular bag that leads to the dislocation of the IOL inside the bag. This results in a decrease of the patient's visual acuity and usually its management requires complex surgical procedures [8]. The risk factors for this condition include clinical conditions that cause zonular weakness such as pseudoexfoliation, connective tissue disorders, uveitis, retinitis pigmentosa, high myopia, and patients who underwent vitreoretinal surgery. Bag dislocation in these situations usually occurs a long time after cataract surgery, with a mean interval of 6.9-8.5 years [9-11]. In 
silico studies shall help understand the mechanics of the IOL dislocation, and study the parameters that could influence this complication, due to its increasing incidence following the increase of procedures performed to remove cataracts [12-14]. However, only a few computational studies on the crystalline lens are found in literature and to the authors' knowledge there are none addressing the IOL dislocation. Previously published works had different purposes, such as understanding the mechanisms of the accommodation process in healthy or presbyopic eyes [15-17], or computationally estimating mechanical properties of the human crystalline lens $[13,18]$.

In fact, building reliable models of the eye is not straightforward because of its complex geometry, together with the lack of information on tissue properties. There are also limited clues on the biomechanics of eye degeneration [19]. The human eye lens complex is composed by four main components: the capsular bag (or capsule), the crystalline lens (composed by the cortex and the nucleus), the zonular fibers (or zonules), and the ciliary body. This complex is of extreme importance in the human visual system since it allows the eye to focus on objects, i.e., to accommodate. The crystalline lens is a transparent and flexible structure enclosed inside a thin membrane with a thickness of about $10 \mu \mathrm{m}$, even though Fisher and Pettet [20] showed it slightly changes with age and position, the capsular bag, and is connected to the ciliary body by the zonular fibers, at the zonular lamella. The zonules are known to be separated in three distinct groups, all around the circumference of the lens: the anterior, the equatorial, and the posterior group. They are considered as suspensory ligaments which not only hold the lens in position, but also reshape it during accommodation.

In addition, the mechanical loading depends on the state of accommodation (accommodated/unaccommodated), and a few theories about accommodation have arisen and were not always in concordance. The most popular one was the Helmholtz's theory [21] that stated that in the unaccommodated (or disaccommodated) state, the lens was held in a state of radial tension by all the zonules. When beginning the process of accommodation, the ciliary body contracted leading to a reduction of tension in the zonular fibers and to an increase of curvature of lens surfaces and optical power, until reaching the fully accommodated state. Opposed to Helmholtz's, stood Schachar's theory [22] stating that when going from the unaccommodated to the accommodated state, the contraction of the ciliary body lead to an increase of tension in the zonular fibers, i.e., stating that the zonules had a direct effect on this process. From a mechanical point of view, Helmholtz claimed that the stress-free state of the lens complex was when it stood in an accommodated state, with no stresses on the zonular fibers, whereas Schachar stated that this stress-free state occurred in the unaccommodated state. Understanding the mechanisms of accommodation was very relevant to study complications regarding the crystalline lens and its associated structures. The main complication to be discussed in this work usually occurs in late adult life, when the crystalline lens loses transparency and becomes opaque-it is said to become a cataract. This results in the reduction of the transmission of light and in scattering light, leading to blurred vision, and in the extreme to blindness, when not treated in time. Recently, the most common solution available is the complete removal of the cataract, i.e., of the crystalline lens, followed by an implant of an IOL in the capsular bag to replace it, leaving the eye in a pseudophakic state $[12,22,23]$.

Assuming the mechanical environment plays an important role in the capsular failure and in the IOL performance, the main objective of this work was to propose a mechanical model of the IOL-capsular bag complex after cataract surgery. Knowing that late IOLcapsular bag dislocation was one of the most serious problems after this procedure, the goal was to study the stress in the capsular bag and in the zonular fibers through a suitable Finite Element (FE) model, to understand when did the zonules break and lead to the dislocation of the whole complex. The development of such a model would allow the assessment of the impact of different variables in the capsular and zonular stresses. In clinical practice, this could translate into changes in intraoperative maneuvers, like the type and size of capsulotomy opening created, or selection of a specific IOL material, design, 
or positioning in the capsular bag that would minimize the risk of a late in-the-bag-IOL dislocation. Meaningful parameters, as the diameter of the capsulotomy and the materials of the IOL were studied to assess their influence on this process and to seek the best configuration to avoid this complication with the IOL.

\section{Materials and Methods}

In this work, an axisymmetric FE model of the crystalline complex during the process of accommodation under cataract surgery is developed. This axisymmetric model, built in Abaqus ${ }^{\circledR}$ (Dassault Systèmes Simulia Corp., Johnston, RI, USA), approaches the threedimensional-like (3D) problem with lower computational complexity. To this end, the model here presented uses a simple cross-section of the human lens, since the loading conditions are considered axially symmetric.

\subsection{Geometry}

The first model built in Abaqus represented the capsular bag, the cortex, the nucleus and the equatorial zonules of the human lens complex, as shown in Figure 1 (upper schematic). The crystalline, i.e., the cortex and nucleus, had both a perfect ellipsoid shape, with the coordinates of the major ( $R_{\text {nucleus }}$ and $R_{\text {lens, }}$, for the nucleus and cortex, respectively) and minor axis (corresponding to the thickness of the lens, $\mathrm{T}_{\text {lens }}$, and to the thickness of the nucleus, $\mathrm{T}_{\text {nucleus }}$ ) adapted from the radii used by Lanchares and co-workers in their study [18]. Based in a linear regression originally proposed by Burd co-workers it was also possible to compute the radius of the ciliary body $\left(\mathrm{R}_{\text {total }}\right)$, and consequently to compute the length of the equatorial zonules $\left(R_{\text {zon }}\right)$ to be used in this model [16]. The capsular bag had the same outline as the cortex, to fit perfectly around it. The cortex and nucleus were modelled as a homogeneous solid, whereas the capsular bag and the zonules as membranes, with constant thicknesses of $10 \mu \mathrm{m}$ [23] and $40 \mu \mathrm{m}$ [24], respectively.

The following models of pseudophakic eyes after cataract surgery, had an IOL replacing the cortex and nucleus of the crystalline after their extraction, and differed in their geometry in the diameter of the capsulorhexis $\left(\Phi_{\mathrm{CCC}}\right)$. As Langwińska-Wośko and coworkers have proved that a smaller diameter had a greater impact on the development of some complications after surgery, such as posterior capsular opacification, it was interesting to see if their diameter could also have an impact on the IOL-capsular bag dislocation [25]. For this purpose, pseudophakic eyes with small and large capsulorhexis were modeled, i.e., with $72.73 \%$ and $81.82 \%$ of the diameter of the IOL optic, respectively [25]. Since the models were built axisymmetric, the capsulorhexis could only be a continuous curvilinear capsulorhexis. The cross-section of the lens that was drawn for this axisymmetric model was based on the cross-section geometry of a one-piece IOL (Alcon SN60WF-Novartis ${ }^{\circledR}$ from Alcon, Fort Worth, TX, USA) described by Sheehan (Figure 1, below) [26]. $\mathrm{R}_{\text {optic }}$ corresponded to the radius of the optic of the lens, $\mathrm{T}_{\text {optic }}$ to the thickness of the optic, and $\mathrm{T}_{\text {haptic }}$ to the thickness of the haptics. In Figure 2, it is possible to observe the three-dimensional like problem, resulting from the revolution of the drawn sections in Figure 1 around their axis of symmetry. 


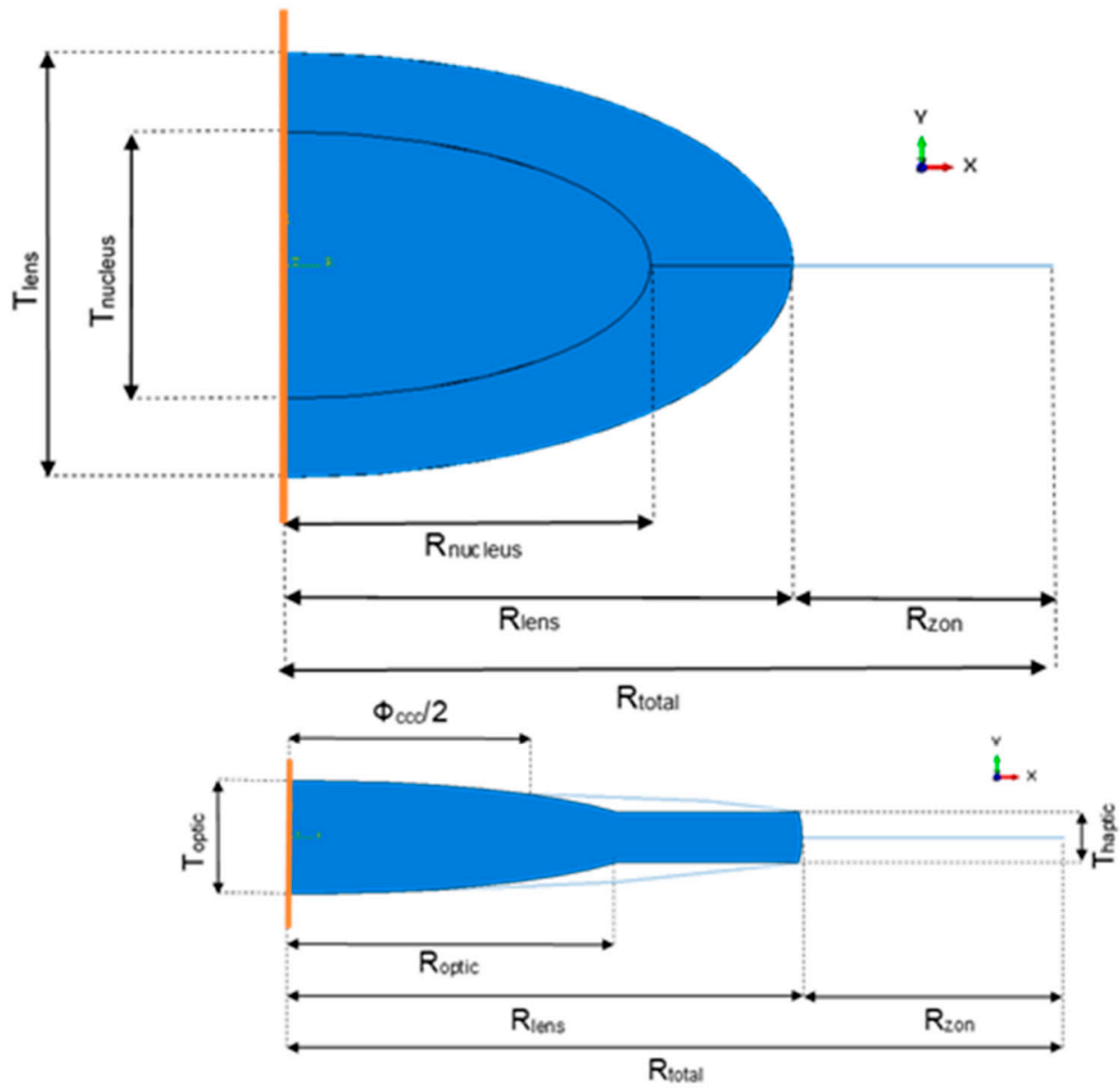

Figure 1. Upper schematic-Geometry of the eye (nucleus, cortex, capsular bag, and zonules) before cataract surgery. The orange line represents the axis of symmetry. Lower schematic-Geometry of the pseudophakic eye (intraocular lens (IOL), capsular bag, and zonules) after cataract surgery. The orange line represents the axis of symmetry.

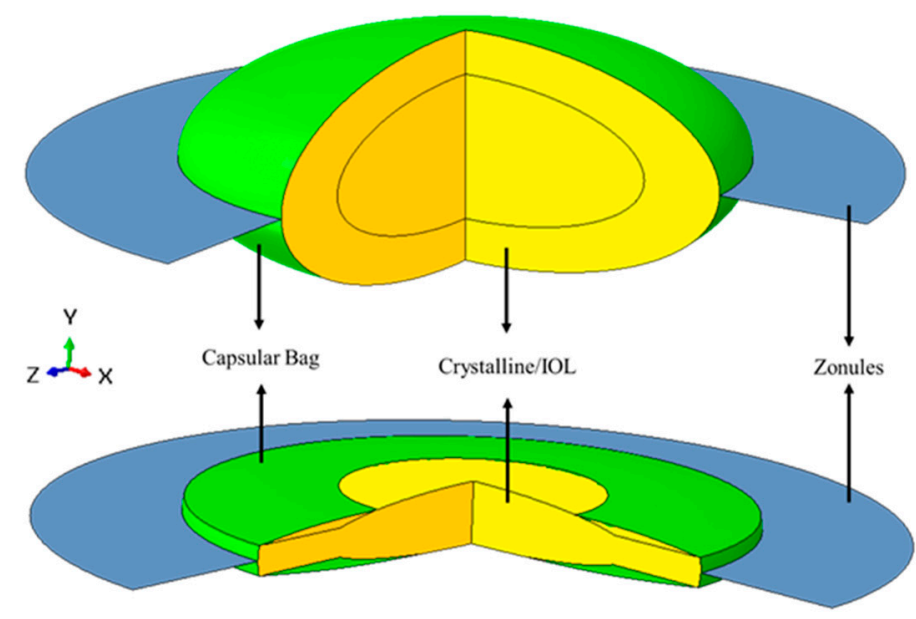

Figure 2. Example of the 3D-like model of the crystalline complex before and after cataract surgery. Figures obtained after revolving the drawn section around the y-axis (symmetry axis) over 270 degrees. Green: capsular bag; Blue: zonules; Yellow: Crystalline/IOL. 


\subsection{Material Properties}

Based on previous studies, all the materials of the human crystalline were defined as linear isotropic and quasi-incompressible. The Young's moduli and Poisson's ratio of the cortex and the nucleus were based on the study of Wang and co-workers [27] and the capsular bag and zonules were based on Weeber and van der Heijde [17]. All of these values are depicted in Table 1. Three different materials were tested for the one-piece IOL, hydrophilic acrylic, hydrophobic acrylic, and poly(methyl methacrylate) (commonly known as PMMA), since they are the most recently used. The mechanical properties of those materials are based on the experimental study performed by Bozukova and co-workers [28].

Table 1. Mechanical properties of the crystalline complex before and after surgery $[16,28,29]$.

\begin{tabular}{ccc}
\hline & Young's Modulus (MPa) & Poisson's Ratio \\
\hline Cortex & 0.0037 & 0.47 \\
Nucleus & 0.0006 & 0.47 \\
Capsular bag & 1.5 & 0.47 \\
Zonules & 0.35 & 0.47 \\
Hydrophilic acrylic & 3.911 & 0.39 \\
Hydrophobic acrylic & 5.829 & 0.39 \\
PMMA & 3000 & 0.39 \\
\hline
\end{tabular}

\subsection{Finite Element Modelling}

The FE model was built with different types of elements, depending on the region: (i) 4-node bilinear axisymmetric quadrilateral elements (CAX4) in the cortex (4644 elements) and nucleus (2648 elements); (ii) 2-node linear axisymmetric membrane elements (MAX1) in the capsular bag (200 elements) and zonules (44 elements); and (iii) finally, the IOL was discretized with 4-node bilinear axisymmetric quadrilateral with reduced integration with hourglass control elements (CAX4R) and 3-node linear axisymmetric triangle elements (CAX3), to ensure a good geometrical fit (1338 elements).

This FE mesh configuration was achieved after a mesh convergence study. Quadratic elements were initially tested, but comparative simulations performed with linear and quadratic elements converged for the same size of element (Figure 3), despite the higher number of nodes on the quadratic elements model. Given that outcome, to decrease complexity and computational weight, all of the following simulations were carried out with linear elements.

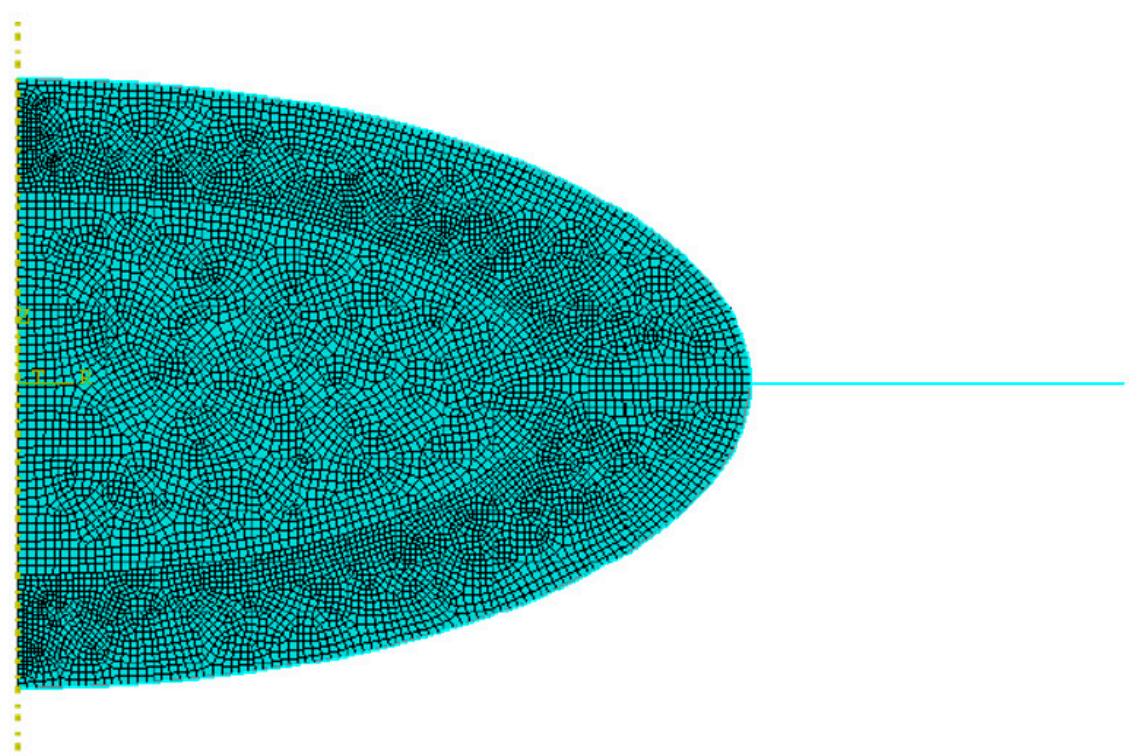

Figure 3. Final Finite Element (FE) mesh employed in this study. 
The accommodation process was first simulated using the model of accommodation proposed by Helmholtz [18], with the accommodated state of the lens being the stress-free state of the model. Then, to mimic the disaccommodation of the crystalline, an outward displacement of $0.5 \mathrm{~mm}(\delta)$ was applied at the tip of the equatorial zonule, where it should be anchored in the ciliary body, with a maximal change in diameter between 1 and $1.2 \mathrm{~mm}$ [27]. Furthermore, for the pseudophakic eye, to simulate the centripetal force towards the center of the opening of the capsulorhexis, a concentrated traction force (TF) was applied at its edge. An important aspect to highlight in axisymmetric models was that prescribed nodal loads or reaction forces were the total values of these loads or forces, integrated along the circumference, around the axis of symmetry. Additionally, there were automatic boundary conditions defined on the axis of symmetry (Y-axis) that constrained the nodes lying on it in the $\mathrm{X}$-direction, i.e., they can only move up- or downward. Considering that the only part that needed to be manually constrained was the equatorial zonule, that could only be moved in the X-direction. This is valid for all the models, before and after cataract surgery. The boundary conditions and load-cases/displacement applied to the models are displayed in Figure 4. Since no in vivo values for this force were found in the literature, few cases were studied, with forces ranging from zero to values higher than the reaction force at the zonules. The interactions occurring between the capsular bag and the crystalline cortex were based on the assumptions proposed by Bassnett and co-workers: the attachment of the capsular bag and the crystalline cortex is strong enough to resist all forces created during the process of accommodation, leading to a tie between both of these components (i.e., a rigid link between all the degrees of freedom of these components) [29]. Since the capsular bag sealed itself to the IOL after surgery, the same tie constraint was also considered between the IOL and the capsule [30,31].
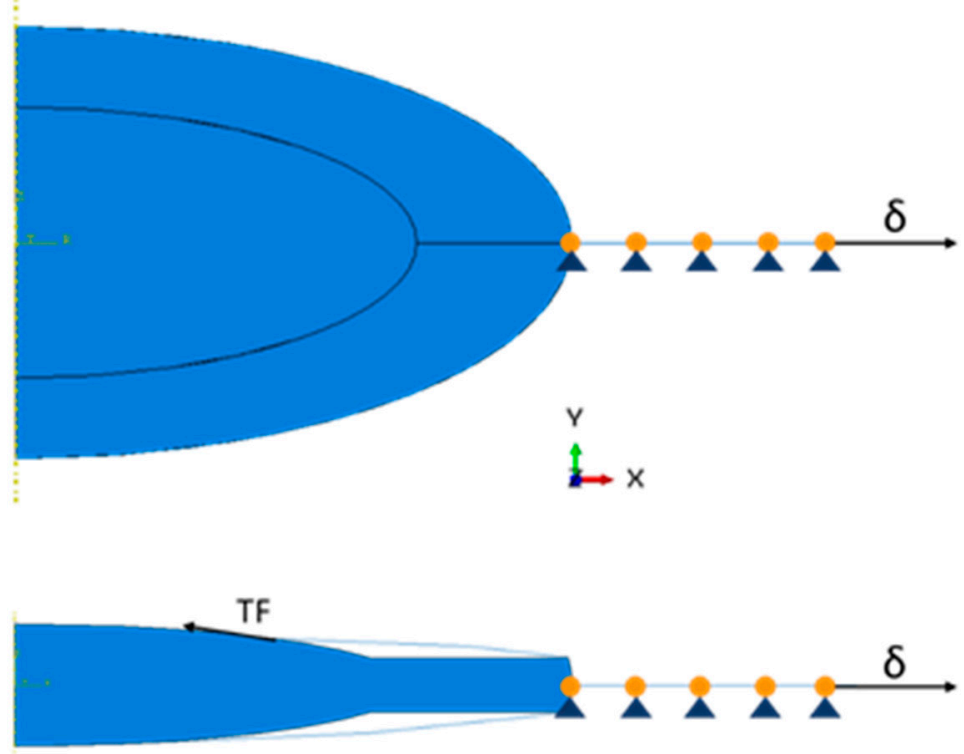

Figure 4. Displacement and loads applied to the models. Upper schematic-only the radial displacement $(\delta)$ is applied at the tip of the zonules for the complete crystalline model. Lower schematic-an additional traction force (TF) is applied at the edge of the capsulorhexis for the pseudophakic models.

With the purpose of studying stresses in the capsular bag, in the zonules and in the IOLs, the change in length in the radial direction (in the $X$ direction) and the resulting forces at the tip of the zonules (RF), a set of 25 models of pseudophakic eyes were built. Since no such FE models were found in the literature, it was important to study the variation and influence of a few parameters, such as the diameter of the capsulorhexis, the IOL material, and the traction force exerted at the opening of the capsulorhexis, on the model. Considering the eye before surgery as M1, the features of all the other models are 
summarized in Table 2. For the traction force, since there was no available information on its values in vivo, some hypothetic values were tested based on the resulting force at the tip of the zonules when no traction force was applied, with smaller, equal, or higher values than this RF.

Table 2. Description of the pseudophakic models discussed in this work, differing in the diameter of their capsulorhexis (mm), their IOL materials and traction forces (TF) at the opening of the capsulorhexis. "RF M 2.1", in model 6.1, means that the reaction forces (RF) of model 2.1 were used.

\begin{tabular}{cccc}
\hline Model & Фccc $(\mathbf{m m})$ & IOL Material & TF (N) \\
\hline M 2.1 & 4 & Hydrophilic acrylic & 0 \\
M 2.2 & 4 & Hydrophobic acrylic & 0 \\
M 2.3 & 4 & PMMA & 0 \\
M 3.1 & 4.5 & Hydrophilic acrylic & 0 \\
M 4.1 & 4 & Hydrophilic acrylic & 0.7 \\
M 5.1 & 4.5 & Hydrophilic acrylic & 0.7 \\
M 6.1 & 4 & Hydrophilic acrylic & RF M 2.1 \\
M 8.1 & 4 & Hydrophilic acrylic & 0.15 \\
\hline
\end{tabular}

\subsection{Numerical/Experimental Validation}

After complete disaccommodation of the complete crystalline, it was possible to compare the change in length in the radial direction $\left(\delta_{R}\right)$ and in total lens thickness $\left(\delta_{\mathrm{T}}\right)$, i.e., in the $X$ and in the $Y$ direction, respectively. The lens had an increase of about $0.35 \mathrm{~mm}$ in the radial direction, corresponding to approximatively $8 \%$ of its initial diameter, very close to the change of $7 \%$ stated by Weeber and van der, and a decrease of $0.25 \mathrm{~mm}$ in lens thickness [17]. This value of $\delta_{R}$ was higher than the one found by Burd and co-workers of $0.29 \mathrm{~mm}$ [16], but was very close to the one found by Lanchares and co-workers of $0.32 \mathrm{~mm}$ [18]. Regarding the lens thickness $\delta_{\mathrm{T}}$, Dubbelman and co-workers and Lanchares and co-workers had output values of 0.33 and $0.39 \mathrm{~mm}$ respectively, higher values than what was calculated in the current study [18,32].

The maximum principal stress distribution in the capsular bag after total disaccommodation, showed a range of stresses going from $6.9 \times 10^{-2}$ to $1.6 \times 10^{-1} \mathrm{MPa}$, that were of the same order than the ones from Lanchares and co-workers that went from $5.3 \times 10^{-2}$ to $1.6 \times 10^{-1} \mathrm{MPa}$ [18]. The distribution of stress was slightly different, since that reference study used a variable thickness of the capsule, with the maximum value corresponding to the posterior pole associated with the lower thickness [18]. Taking the maximum logarithmic strain of the capsular bag, it was possible to compute its strain, $\lambda_{\text {principal, }}$, that was equal to $7.66 \%$. This value was very similar to the $7.28 \%$ strain computed by Lanchares and co-workers [18]. Another parameter that could be compared with the literature, was the resulting force in the zonules after their displacement. The resulting force computed at the tip of the zonules had a magnitude of $0.071 \mathrm{~N}$, that was in the same range as the $0.078 \mathrm{~N}$ from Lanchares and co-workers and the $0.08-0.1 \mathrm{~N}$ from Burd and co-workers [16,18]. Therefore, the model presented here was considered acceptable despite the simplifications assumed during its construction, such as its geometry and the constant thickness of the capsule. The validation outputs are summarized in Table 3.

Table 3. Summary of literature values for model validation $[15,17,31]$. The initial radius of the lens ( $\left.\mathrm{R}^{\text {lens }}\right)$ as well as its initial thickness $\left(\mathrm{T}_{\text {lens }}\right)$ are also shown.

\begin{tabular}{ccccccc}
\hline Model & $\mathbf{R}_{\mathbf{l e n s}}(\mathbf{m m})$ & $\mathbf{T}_{\text {lens }}(\mathbf{m m})$ & $\boldsymbol{\delta}_{\mathbf{R}}(\mathbf{m m})$ & $\boldsymbol{\delta}_{\mathbf{R}} \mathbf{( \% )}$ & $\boldsymbol{\delta}_{\mathbf{T}}(\mathbf{m m})$ & $\boldsymbol{\delta}_{\mathbf{T}} \mathbf{( \% )}$ \\
\hline Membrane Capsule & 4.30 & 3.60 & 0.35 & 8.1 & 0.25 & 6.9 \\
Shell Capsule & 4.30 & 3.60 & 0.35 & - & 0.25 & 0.33 \\
Dubbelman et al., 2003 & - & 3.63 & - & 6.6 & 0.39 & 1 \\
Lanchares et al., 2012 & 4.88 & 3.57 & 0.32 & 7.6 & - \\
Burd et al., 2001 & 3.83 & - & 0.29 & - & -1 \\
\hline
\end{tabular}




\section{Results}

For each model, the von Mises stress in the zonules $\left(\mathrm{S}_{\mathrm{Z}}\right)$, in the capsular bag $\left(\mathrm{S}_{\mathrm{CB}}\right)$ and in the IOL $\left(\mathrm{S}_{\mathrm{IOL}}\right)$, were assessed, as well as the radial displacement in the $\mathrm{X}$-direction $\left(\delta_{\mathrm{r}}\right)$ and the resulting force at the tip of the zonules $\left(R F_{Z}\right)$. All of the models built had the purpose of letting us compare different models for pseudophakic eyes, with different diameters of capsulorhexis, different materials for the different one-piece IOL, and with different traction forces towards the center of the capsulorhexis, that simulated part of the process of fibrosis.

\subsection{Post-Surgery}

A comparison between the eye before (model M1) and after surgery with a small capsulorhexis, a hydrophilic acrylic IOL, and no traction force (model M 2.1) was possible and is shown in Figure 5. The average values of stresses, radial displacement, and resulting force are shown in Table 4 for both models. The values of stresses in the zonular fibers are almost the double from M1 to M 2.1, going from an average value of $4.7 \times 10^{-2}$ to $8.9 \times 10^{-2} \mathrm{MPa}$, and from the crystalline to the IOL it substantially increased 30 times from an average value of $1.9 \times 10^{-4}$ to $5.8 \times 10^{-3} \mathrm{MPa}$, whereas in the capsular bag the values decreased, going from average values of $8.0 \times 10^{-2}$ to $1.9 \times 10^{-3} \mathrm{MPa}$. The radial displacement in M 2.1, equal to $7.3 \times 10^{-3} \mathrm{~mm}$ was almost fifty-times smaller than in M1, $3.5 \times 10^{-1} \mathrm{~mm}$. Another value that increased from M1 to M 2.1 was the resulting force at the tip of the zonular fibers, with computed values of $7.1 \times 10^{-2}$ and $12.8 \times 10^{-2} \mathrm{~N}$ for the model before and after surgery, respectively. All of these outcomes were expected with the increase of stiffness of the three IOL materials in comparison with the natural cortex and nucleus of the crystalline. With a maximum of $1.5 \times 10^{-1} \mathrm{MPa}$, the higher stress in $\mathrm{M} 1$ was located on the capsular bag, whereas in model M 2.1 this was located on the zonular fibers with a value of $9.7 \times 10^{-2} \mathrm{MPa}$.
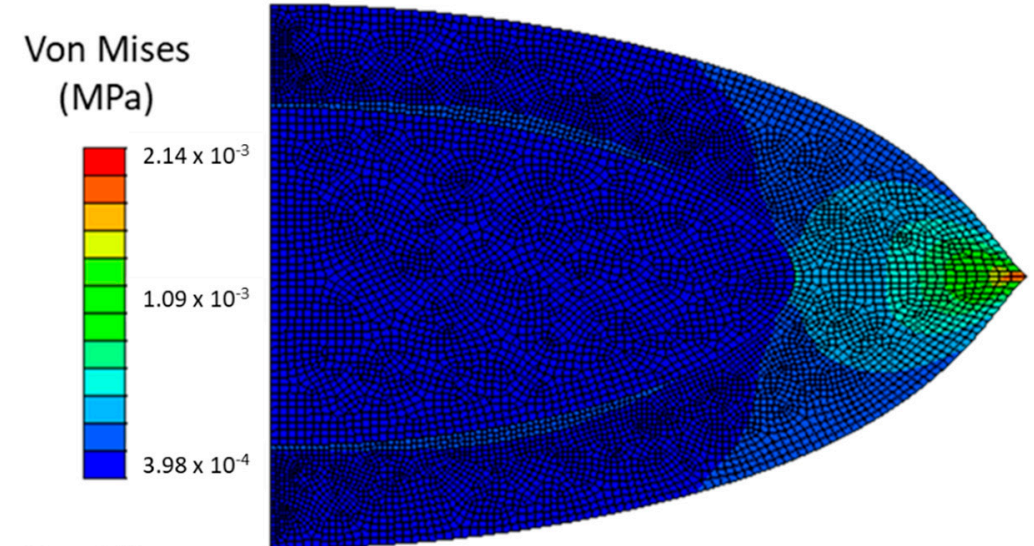

Von Mises

(MPa)

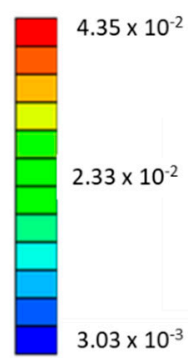

Figure 5. Upper schematic-von Mises stresses in in the crystalline. Lower schematic-von Mises stresses in a pseudophakic eye (IOL), after cataract surgery with a $4 \mathrm{~mm} \Phi_{\mathrm{CCC}}$ and a hydrophilic acrylic IOL, following ciliary body relaxation. 
Table 4. Average values of von Mises stresses in the zonular fibers, capsular bag, and crystalline/IOL, as well as radial displacement and resulting force at the tip of the zonular fibers for the models M1 and $\mathrm{M} 2.1$.

\begin{tabular}{ccc}
\hline Model & Variables & Average Value \\
\hline & $\mathrm{S}_{\mathrm{Z}}(\mathrm{MPa})$ & $4.7 \times 10^{-2}$ \\
& $\mathrm{~S}_{\mathrm{CB}}(\mathrm{MPa})$ & $8.0 \times 10^{-2}$ \\
$\mathrm{M} 1$ & $\mathrm{~S}_{\mathrm{IOL}}(\mathrm{MPa})$ & $1.9 \times 10^{-4}$ \\
& $\delta_{\mathrm{r}}(\mathrm{mm})$ & $3.5 \times 10^{-1}$ \\
& $\mathrm{RF}_{\mathrm{Z}}(\mathrm{N})$ & $7.1 \times 10^{-2}$ \\
& $\mathrm{~S}_{\mathrm{Z}}(\mathrm{MPa})$ & $8.9 \times 10^{-2}$ \\
& $\mathrm{~S}_{\mathrm{CB}}(\mathrm{MPa})$ & $1.9 \times 10^{-3}$ \\
& $\mathrm{~S}_{\mathrm{IOL}}(\mathrm{MPa})$ & $5.8 \times 10^{-3}$ \\
& $\delta_{\mathrm{r}}(\mathrm{mm})$ & $7.3 \times 10^{-3}$ \\
& $\mathrm{RF}_{\mathrm{Z}}(\mathrm{N})$ & $12.8 \times 10^{-2}$ \\
\hline
\end{tabular}

\subsection{Influence of IOL Stiffness}

To study the influence of the stiffness of the IOL in the pseudophakic eye, a comparison between models M 2.1., M 2.2., and M 2.3. was performed. These three models had the same $\Phi_{\mathrm{CCC}}$ and TF, equal to zero, but differed in their IOL material and consequently, in their stiffness. The results drawn in this section can be drawn for every other three sets of models, that only differ in IOL stiffness.

Since the PMMA lens was at least 500-timed stiffer than both acrylic lenses, the expected outcome would be that it would support more stress in it with a consequent decrease of stress in the capsular bag. This phenomenon is visible in Figure 6, where model M 2.3 had a different stress distribution, with the stress more distributed through the IOL haptic, than M 2.1 and M 2.2, with a maximum value of $4.4 \times 10^{-2} \mathrm{MPa}$, at the intersection between the capsular bag, the IOL and the insertion of the zonule. Whereas the general decrease of stress in the capsular bag was very slight between the hydrophilic and the hydrophobic acrylic lens, with average values ranging from $1.9 \times 10^{-3}$ to $1.4 \times 10^{-3} \mathrm{MPa}$ respectively, an accentuated change was clearly seen in model $M$ 2.3., with an average value of $8.9 \times 10^{-6} \mathrm{MPa}$, being 150-times lower than in M 2.1 or M 2.2. With the increase of stiffness in the IOL, more resistance to the equatorial pull in the zonules in the materials would be encountered, leading to a steep decrease of radial displacement in the IOL, with the one in model M 2.3, $1.2 \times 10^{-5} \mathrm{~mm}$, being 6000-times higher than in the other two models, $7.3 \times 10^{-3} \mathrm{~mm}$ for the hydrophilic and $5.0 \times 10^{-3} \mathrm{~mm}$ for the hydrophobic acrylic lens. In Table 5, the values with the biggest noticeable changes are depicted.

Table 5. Average values of von Mises stresses in capsular bag and radial displacement at the tip of the zonular fibers for the models M 2.1., M 2.2., and M 2.3.

\begin{tabular}{ccc}
\hline Model & Variables & Average Value \\
\hline \multirow{2}{*}{ M 2.1} & $\mathrm{~S}_{\mathrm{CB}}(\mathrm{MPa})$ & $1.9 \times 10^{-3}$ \\
& $\delta_{\mathrm{r}}(\mathrm{mm})$ & $7.3 \times 10^{-3}$ \\
M 2.2 & $\mathrm{~S}_{\mathrm{CB}}(\mathrm{MPa})$ & $1.4 \times 10^{-3}$ \\
& $\delta_{\mathrm{r}}(\mathrm{mm})$ & $5.0 \times 10^{-3}$ \\
M 2.3 & $\mathrm{~S}_{\mathrm{CB}}(\mathrm{MPa})$ & $8.9 \times 10^{-6}$ \\
& $\delta_{\mathrm{r}}(\mathrm{mm})$ & $1.2 \times 10^{-6}$ \\
\hline
\end{tabular}




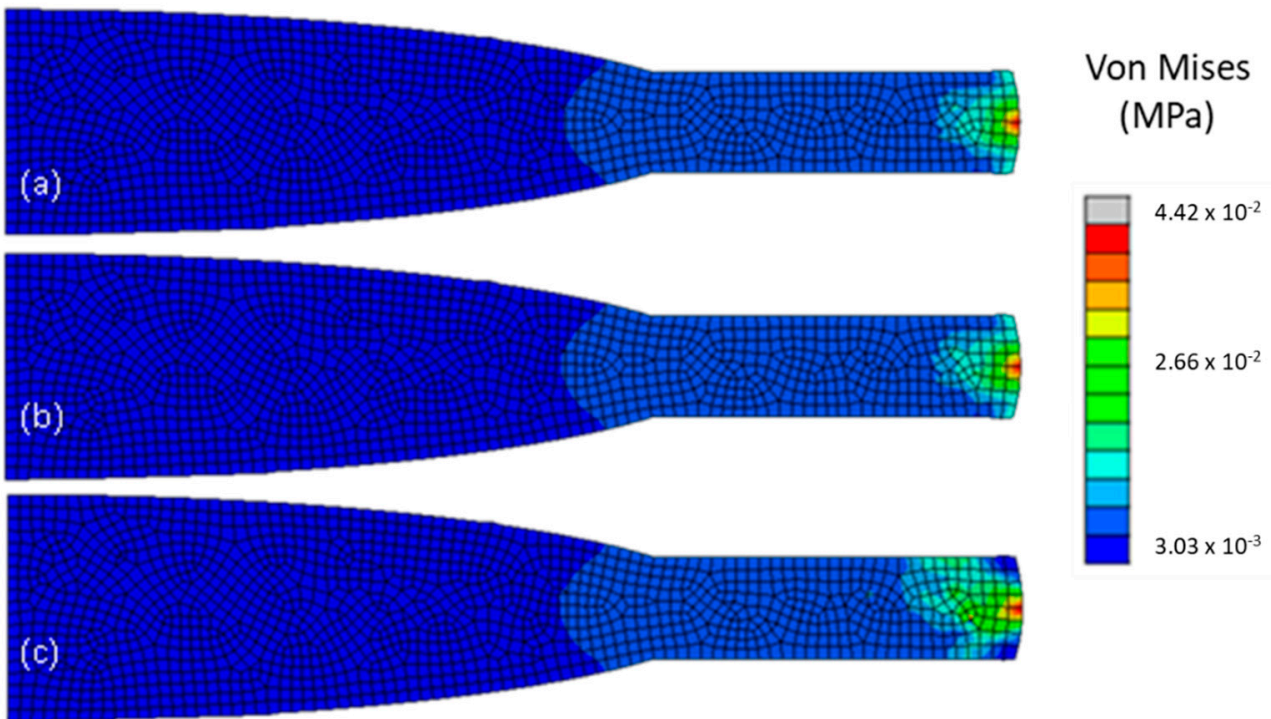

Figure 6. Von Mises stresses (in MPa) in the IOL, for the three models, after cataract surgery with a $4 \mathrm{~mm} \Phi_{\mathrm{CCC}}$ and no traction force, following ciliary body relaxation: (a) model M 2.1. with a hydrophilic acrylic IOL, (b) model M 2.2., with a hydrophobic acrylic IOL, and (c) model M 2.3, with a PMMA IOL.

\subsection{Influence of Traction Force}

To study the influence of the traction force on the capsulorhexis on the pseudophakic eyes, models M 2.1., M 4.1., M 6.1., and M 8.1. were compared, as shown in Figure 7. They all had a one-piece IOL made of hydrophilic acrylic and the same $\Phi_{\mathrm{CCC}}$ but differed in the traction force that was applied. It increased from 0 to $0.15 \mathrm{~N}$, from model M 2.1 to model M 8.1. The outcomes for this parametric study are depicted in Table 5.
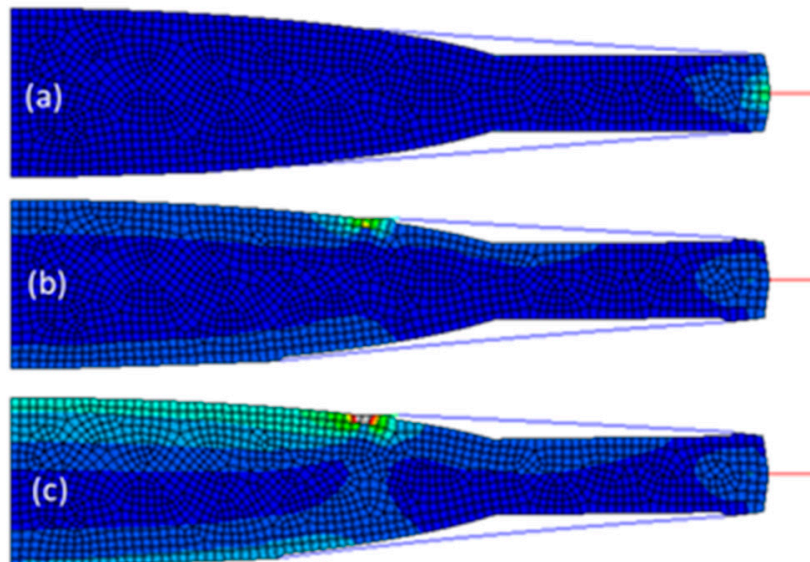

Von Mises

(MPa)
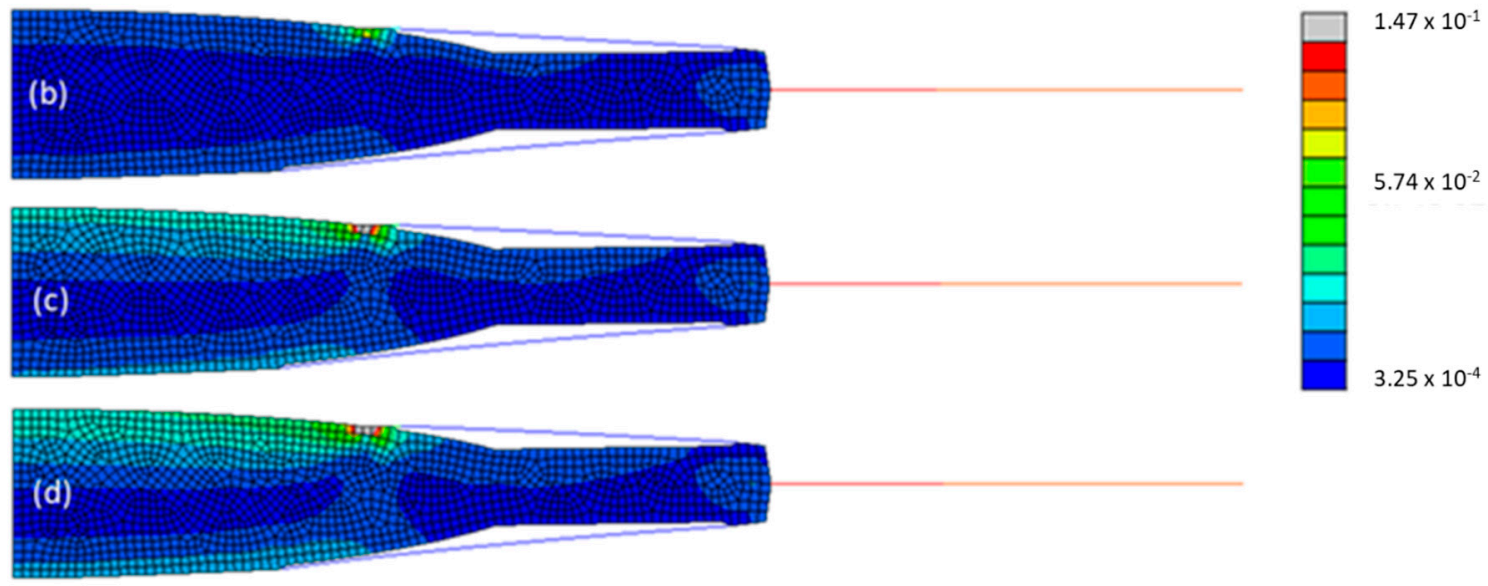

Figure 7. Von Mises stresses in all the components of the four models, after cataract surgery with a $4 \mathrm{~mm} \Phi_{\mathrm{CCC}}$ and a hydrophilic acrylic IOL, following ciliary body relaxation. (a) model M 2.1., (b) model M 4.1., (c) model M 6.1., and (d) model M 8.1.

With the increase of the traction force, no change in stress in the zonules nor in the resulting force at their tip was noticeable, with an average value of $8.9 \times 10^{-2} \mathrm{MPa}$ for the stress and $12.9 \times 10^{-2} \mathrm{~N}$ for the resulting force in all models. Overall, the average value 
of stress in the capsular bag increased 3.5 times from the model with no traction force to the one with a traction force of $0.15 \mathrm{~N}$. The stress in the IOL and radial displacement had the greatest change, with the average stress of $1.4 \times 10^{-2} \mathrm{MPa}$ in the IOL of M 8.1, being almost 2.5-times the average stress, which was equal to $5.8 \times 10^{-3} \mathrm{MPa}$ in $\mathrm{M} 2.1$. The radial displacement, $7.3 \times 10^{-3} \mathrm{~mm}$ in $\mathrm{M} 2.1$, was almost three-times higher than the one in $\mathrm{M}$ $8.1,2.8 \times 10^{-3} \mathrm{~mm}$.

\subsection{Influence of Capsulorhexis Diameter}

In this subsection, models M 4.1 and M 5.1 are compared. They had the same material properties and a traction force applied at the opening of the capsulorhexis of $0.07 \mathrm{~N}$ but differed in the diameter of the capsulorhexis. Models M 2.1 and M 4.1 had a small capsulorhexis with a 4-mm diameter and models M 3.1 and M 5.1 had a large one, with a $4.5-\mathrm{mm}$ diameter. There was no noticeably great variation in the average values of stress in all components.

\section{Discussion}

Comparing the human crystalline complex with a pseudophakic eye, a decrease of stress in the capsular bag and of radial displacement of the new complex was observed, with a consequent increase of resulting force and stress in the zonules, as well as an increase in stress in the IOL. These outcomes could be compared with the influence of the stiffness of the IOL material through the pseudophakic models, since the biggest difference in the eye after surgery, is the difference in stiffness of the human crystalline compared with the stiffness of all the synthetic IOL materials. The IOLs were at least a thousand-times stiffer than any component of the crystalline, making them support more stress than the crystalline and consequently relieve the stress in the capsular bag. With all of the models undergoing the same displacement of $0.5 \mathrm{~mm}$ at the tip of the zonules, the stiff IOL lead to the zonules having to stretch more to pull the lens and the capsular bag, and consequently putting the zonular fibers in a higher state of stress, with its maximum at the intersection node of the three parts of the model. On one hand, the IOLs gave more support to minimize stress in the capsule and eventually decrease its risk of rupture, but on the other hand it put the zonules into a state of more stress with a higher possibility for them to break and lead to an IOL-capsular bag dislocation. Ideally, to avoid this post-operative complication, a lens with a stiffness closer to the one of the human crystalline would be the best solution to try and maintain the state of stress of the capsular bag and the zonules closer to their original state.

Most of the parameters tested seemed to have influenced the overall behavior of the model. In summary, with the increase of the traction force and of the stiffness of the materials, i.e., their Young's modulus, the stress in the IOL would increase, whereas the stress in the capsular bag and the radial displacement would decrease. With the increase of the diameter of the capsulorhexis, and the traction force equal to zero, the overall stress in the models would not change. The radial displacement would decrease and the resulting force increase, but its low magnitude is not significant. Finally, with the increase of the diameter of the capsulorhexis, all the parameters studied seemed to vary slightly and would have a contrary behavior than the ones described earlier, i.e., the stress in the zonules and in the IOL decreased, as well as the resulting force at the tip of the zonules, whereas the stress in the capsular bag and the radial displacement increased.

It was also important to notice that across all models of pseudophakic eyes, the part that would undergo the least change were the zonules, where no parameter seemed to influence greatly their maximum and average values of stress nor the resulting forces computed at their tips. The maximum variation between the average values of stress was $1 \%$ and between the resulting force was $0.8 \%$.

Comparing only pseudophakic eyes, knowing that the stiffer PMMA IOL was at least 500 -times stiffer than the acrylic lenses, a slight increase of 5\% in average stress of the IOL can be considered negligible. The biggest changes observed while increasing the stiffness 
of the IOL was a big decrease in average capsular stress and in radial displacement. This outcome is comparable with the case studied before, i.e., the substitution of the crystalline with any IOL, where an increase in IOL stiffness would give more support to the capsular bag and minimize its average stress. In the zonules, no change in maximum and average values of stress was observed, nor change in the resulting force at their tip.

The increase of the traction force at the opening of the capsulorhexis showed a big influence in the distribution of stress in all of the models, increasing the stress in the IOL (principally in its optic) and decreasing the radial displacement, substantially. Even without knowing the in vivo values of the traction force at the edge of the capsulorhexis, it was relevant to study it to understand if the models built in this work were sensible to its variation. It was expected to observe an increase in average stress in the capsular bag and in the IOL, since these two parts were subjected to an increasing load in the opposite direction from that of the ciliary body relaxation. Furthermore, the natural fibrosis growing on the edge of the capsulotomy is understood to produce a centripetal force towards the center of its opening. This force is greater than the force exerted by the zonules, i.e., it could lead to the rupture of the zonular fibers and cause late IOL-capsular bag dislocation $[14,33]$. However, in this work, it was shown that an increase of this centripetal force, i.e., the traction force that was applied, did not influence the average stress in the zonules, even when it was higher than the resulting force in the zonules.

A small and large capsulorhexis, with $72.73 \%$ and $82.83 \%$ of the diameter of the IOL optic were compared and seemed to have a slight impact on the stresses of the model. With a smaller capsulorhexis it seemed possible to relieve some of the stress in the capsular bag and eventually avoid its rupture, with a consequent IOL dislocation. This outcome seemed to be contrary to the one given by Gimbel and co-workers that stated that a smaller capsulorhexis lead to a higher probability of incidence of IOL-capsular bag dislocation, because it developed more fibrosis than a large capsulorhexis [34]. In this work, it was assumed that both large and small capsulorhexis had suffered the same level of natural fibrosis and that the resulting traction force on its edge was the same in both cases. For that same traction force, the smaller capsulorhexis seemed more favorable in giving support to the capsular bag.

The stress in the zonules doubled after surgery whereas in the capsular bag the same outcomes decreased significantly in most of the models. This is related to the increased stiffness in any IOL compared with the crystalline. The fact that the IOLs have a stiffness at least a thousand-times higher than the human crystalline lead to the IOLs supporting the capsular bag, shielding it from the native stress distribution. No parameter studied here lowered the general state of stress in the zonules post-surgery. The simplified geometry of the natural human crystalline lens model could have an impact on the distribution of stress in the capsular bag, but the outcomes of the pseudophakic eye models were not significantly altered. This is most likely associated with the shape alteration of the capsule after surgery, shrinking itself to the IOL.

Since this work presented an axisymmetric model, the IOLs were simplified to represent perfectly axisymmetric lenses, which is not the real configuration of the lens. The geometry of the IOLs could be improved in future works, as in 3D models with different types of asymmetric IOLs (one-piece, three-piece, or plate IOLs). Moreover, additional parameters could be studied to understand their influence in the overall stress along the different sections of the models, i.e., to study the influence of the gravity, the positioning of the IOL, the type of capsulotomy performed, and different densities of zonules (to mimic zonular dehiscence). In this study, the solid and fluid problems were completely decoupled, and the influence of the surrounding aqueous humor was not considered. Considering that the stresses measured in the crystalline lens and in the IOL are larger than those generated in the aqueous humor, a complementary computational fluid dynamics (CFD) study could also be performed to study the influence of the aqueous humor over the solid part of the model (CFD-FE coupled simulation) [35]. In vivo values of the tensile strength of the zonules and the capsular bag would also have a great impact on the models, since 
it would be possible to replicate more realistic behaviors in these materials and to know when they would tear and lead to an IOL dislocation. This lack of in vivo information about the materials' properties is a major limitation for works in this field, so the necessary parameters were sourced from in silico studies of the complete crystalline complex.

\section{Conclusions}

In this work, a human crystalline lens and its associated structures were discretized to an axisymmetric FE model, before and post-IOL implantation. As so, the models included the capsular bag and the zonular fibers, and pseudophakic eyes with a one-piece IOL. A radial displacement was applied at the tip of the zonular fibers to mimic the movement of the ciliary body during the process of disaccommodation.

The major finding from this work is that the variation of parameters in the pseudophakic models showed no significant impact on the zonules. It was important to verify the impact of the increase of the Young's moduli of the IOLs, different diameters of capsulorhexis and increased traction force on the zonules, since their rupture is the main precursor of IOL-capsular bag dislocation. Since no significant alterations were observed, it is possible to speculate that a more realistic geometry of an IOL could have a greater influence on the outcomes of the simulations.

To the authors' best knowledge, this is the first FE study addressing the eye under cataract surgery. Notwithstanding the model limitations, the outcomes presented here can be an important complement for the existing studies on the crystalline complex, both on clinical practice and biomechanics research.

Author Contributions: Conceptualization, B.F., A.P.G.C., F.J.R. and P.R.F.; methodology, M.T.C. and A.P.G.C.; software, M.T.C.; validation, B.F., A.P.G.C., F.J.R. and P.R.F.; formal analysis, M.T.C. and A.P.G.C.; investigation, M.T.C.; resources, P.R.F.; data curation, M.T.C.; writing-original draft preparation, M.T.C.; writing-review and editing, A.P.G.C. and P.R.F.; visualization, M.T.C.; supervision, A.P.G.C., F.J.R. and P.R.F.; project administration, F.J.R. and P.R.F.; funding acquisition, A.P.G.C. and P.R.F. All authors have read and agreed to the published version of the manuscript.

Funding: This work was supported by FCT, through IDMEC, under LAETA project UIDB/50022/2020.

Institutional Review Board Statement: Not applicable.

Informed Consent Statement: Not applicable.

Data Availability Statement: Not applicable.

Conflicts of Interest: The authors declare no conflict of interest.

\section{References}

1. Prokofyeva, E.; Wegener, A.; Zrenner, E. Cataract prevalence and prevention in Europe: A literature review. Acta Ophthalmol. 2013, 91, 395-405. [CrossRef] [PubMed]

2. Bikbov, M.M.; Kazakbaeva, G.M.; Gilmanshin, T.R.; Zainullin, R.M.; Nuriev, I.F.; Zaynetdinov, A.F.; Israfilova, G.Z.; Panda-Jonas, S.; Arslangareeva, I.I.; Rakhimova, E.M.; et al. Prevalence and associated factors of cataract and cataract-related blindness in the Russian Ural Eye and Medical Study. Sci. Rep. 2020, 10, 1-9. [CrossRef] [PubMed]

3. Flaxman, S.R.; Bourne, R.R.A.; Resnikoff, S.; Ackland, P.; Braithwaite, T.; Cicinelli, M. V.; Das, A.; Jonas, J.B.; Keeffe, J.; Kempen, J.; et al. Global causes of blindness and distance vision impairment 1990-2020: A systematic review and meta-analysis. Lancet Glob. Health 2017, 5, e1221-e1234. [CrossRef]

4. Oshika, T.; Negishi, K.; Noda, T.; Arai, H.; Inamura, M.; Inoue, Y.; Miyoshi, T.; Fujita, Y.; Miyata, K.; Hasegawa, Y. Prospective assessment of plate-haptic rotationally asymmetric multifocal toric intraocular lens with near addition of +1.5 diopters. BMC Ophthalmol. 2020, 20, 1-9. [CrossRef] [PubMed]

5. Abdalla Elsayed, M.E.A.; Ahmad, K.; Al-Abdullah, A.A.; Malik, R.; Khandekar, R.; Martinez-Osorio, H.; Mura, M.; Schatz, P. Incidence of Intraocular Lens Exchange after Cataract Surgery. Sci. Rep. 2019, 9, 1-7. [CrossRef]

6. Naderi, K.; Gormley, J.; O’Brart, D. Cataract surgery and dry eye disease: A review. Eur. J. Ophthalmol. $2020,30,840-855$. [CrossRef]

7. Al-Halafi, A.M.; Al-Harthi, E.; Al-Amro, S.; El-Asrar, A.A. Visual outcome and complications of pars plana vitrectomy for dislocated intraocular lenses. Saudi J. Ophthalmol. 2011, 25, 187-192. [CrossRef]

8. Gross, J.G.; Kokame, G.T.; Weinberg, D. V. In-the-bag intraocular lens dislocation. Am. J. Ophthalmol. 2004, 137, 630-635. 
9. Lorente, R.; de Rojas, V.; Vazquez de Parga, P.; Moreno, C.; Landaluce, M.L.; Domínguez, R.; Lorente, B. Management of late spontaneous in-the-bag intraocular lens dislocation: Retrospective analysis of 45 cases. J. Cataract Refract. Surg. 2010, 36, 1270-1282. [CrossRef]

10. Hayashi, K.; Hirata, A.; Hayashi, H. Possible Predisposing Factors for In-the-Bag and Out-of-the-Bag Intraocular Lens Dislocation and Outcomes of Intraocular Lens Exchange Surgery. Ophthalmology 2007, 114, 969-975. [CrossRef]

11. Jehan, F.S.; Mamalis, N.; Crandall, A.S. Spontaneous late dislocation of intraocular lens within the capsular bag in pseudoexfoliation patients. Ophthalmology 2001, 108, 1727-1731. [CrossRef]

12. Ong, A.Y.; Shalchi, Z. Outcomes of cataract surgery in pseudoexfoliation syndrome in England: 10-year retrospective cohort study. J. Cataract Refract. Surg. 2021, 47, 165-171. [CrossRef]

13. Wang, K.; Hoshino, M.; Uesugi, K.; Yagi, N.; Pierscionek, B.K. Contributions of shape and stiffness to accommodative loss in the ageing human lens: A finite element model assessment. J. Opt. Soc. Am. A 2019, 36, B116-B122. [CrossRef]

14. Brezna, W.; Dragostinoff, N.; Prinz, M. Human Eye Modeling for Intraocular Lens Design and for Calculating Intraocular Lens Power. IFAC Proc. Vol. 2012, 45, 534-539. [CrossRef]

15. Burd, H.J.; Judge, S.J.; Flavell, M.J. Mechanics of accomodation of the human eye. Vis. Res. 1999, 39, 1591-1595. [CrossRef]

16. Burd, H.J.; Judge, S.J.; Cross, J.A. Numerical modelling of the accommodating lens. Vis. Res. 2002, 42, 2235-2251. [CrossRef]

17. Weeber, H.A.; van der Heijde, R.G.L. Internal deformation of the human crystalline lens during accommodation. Acta Ophthalmol. 2008, 86, 642-647. [CrossRef] [PubMed]

18. Lanchares, E.; Navarro, R.; Calvo, B. Hyperelastic modelling of the crystalline lens: Accommodation and presbyopia. J. Optom. 2012, 5, 110-120. [CrossRef]

19. Han, F.; Li, M.; Wei, P.; Ma, J.; Jhanji, V.; Wang, Y. Effect of biomechanical properties on myopia: A study of new corneal biomechanical parameters. BMC Ophthalmol. 2020, 20, 1-8. [CrossRef]

20. Fisher, R.; Pettet, B. The postnatal growth of the capsule of the human crystalline lens. J. Anat. 1972, 112, 207-214. [PubMed]

21. Southall, J.P.C. Helmholtz's Treatise on Physiological Optics (Volume 3); Dover Publications: New York, NY, USA, 1962; ISBN 0486600165.

22. Schachar, R.A. Cause and treatment of presbyopia with a method for increasing the amplitude of accommodation. Ann. Ophthalmol. 1992, 24, 445-447, 452.

23. Liu, X.; Wang, L.; Du, C.; Li, D.; Fan, Y. Mechanism of lens capsular rupture following blunt trauma: A finite element study. Comput. Methods Biomech. Biomed. Engin. 2015, 18, 914-921. [CrossRef]

24. van Alphen, G.W.H.M.; Graebel, W.P. Elasticity of tissues involved in accommodation. Vision Res. 1991, 31, 1417-1438. [CrossRef]

25. Langwinska- Wosko, E.; Broniek- Kowalik, K.; Szulborski, K. The impact of capsulorhexis diameter, localization and shape on posterior capsule opacification. Med. Sci. Monit. 2011, 17, CR577-CR582. [CrossRef] [PubMed]

26. Sheehan, M.T. Eye Modelling for Personalised Intraocular Lens Design; NUI Galway: Galway, Ireland, 2012.

27. Wang, K.; Venetsanos, D.T.; Wang, J.; Augousti, A.T.; Pierscionek, B.K. The importance of parameter choice in modelling dynamics of the eye lens article. Sci. Rep. 2017, 7, 1-12.

28. Bozukova, D.; Werner, L.; Mamalis, N.; Gobin, L.; Pagnoulle, C.; Floyd, A.; Liu, E.; Stallings, S.; Morris, C. Double-C loop platform in combination with hydrophobic and hydrophilic acrylic intraocular lens materials. J. Cataract Refract. Surg. 2015, 41, 1490-1502. [CrossRef] [PubMed]

29. Bassnett, S.; Missey, H.; Vucemilo, I. Molecular architecture of the lens fiber cell basal membrane complex. J. Cell Sci. 1999, 112 Pt 1, 2155-2165.

30. Ascaso, F.J.; Huerv, V. The History of Cataract Surgery. In Cataract Surgery; InTech: London, UK, 2013; ISBN 978-953-51-0975-4.

31. Auffarth, G.U.; Gerl, M.; Tsai, L.; Janakiraman, D.P.; Jackson, B.; Alarcon, A.; Dick, H.B. Clinical evaluation of a new monofocal IOL with enhanced intermediate function in patients with cataract. J. Cataract Refract. Surg. 2021, 47, 184-191. [CrossRef]

32. Dubbelman, M.; Van Der Heijde, G.L.; Weeber, H.A.; Vrensen, G.F.J.M. Changes in the internal structure of the human crystalline lens with age and accommodation. Vision Res. 2003, 43, 2363-2375. [CrossRef]

33. Hartman, M.; Rauser, M.; Brucks, M.; Chalam, K. V. Evaluation of anterior capsular contraction syndrome after cataract surgery with commonly used intraocular lenses. Clin. Ophthalmol. 2018, 12, 1399-1403. [CrossRef]

34. Gimbel, H. V.; Condon, G.P.; Kohnen, T.; Olson, R.J.; Halkiadakis, I. Late in-the-bag intraocular lens dislocation: Incidence, prevention, and management. J. Cataract Refract. Surg. 2005, 31, 2193-2204. [CrossRef] [PubMed]

35. Fernández-Vigo, J.I.; Marcos, A.C.; Agujetas, R.; Montanero, J.M.; Sánchez-Guillén, I.; García-Feijóo, J.; Pandal-Blanco, A.; Fernández-Vigo, J.Á.; Macarro-Merino, A. Computational simulation of aqueous humour dynamics in the presence of a posteriorchamber versus iris-fixed phakic intraocular lens. PLoS ONE 2018, 13, e0202128. [CrossRef] [PubMed] 